\title{
Impact of early intervention on outcome following mild head injury in adults
}

\author{
J Ponsford, C Willmott, A Rothwell, P Cameron, A-M Kelly, R Nelms, C Curran
}

J Neurol Neurosurg Psychiatry 2002;73:330-332

Background: The impact of mild head injury is variable and determinants of outcome remain poorly understood. Results of previous intervention studies have been mixed.

Objectives: To evaluate the impact on outcome of the provision of information, measured in terms of reported symptoms, cognitive performance, and psychological adjustment three months postinjury.

Methods: 202 adults with mild head injury were studied: 79 were assigned to an intervention group and were assessed one week and three months after injury; 123 were assigned to a non-intervention control group and were seen at three months only. Participants completed measures of preinjury psychological adjustment, concurrent life stresses, post-concussion symptoms, and tests of attention, speed of information processing, and memory. Subjects seen at one week were given an information booklet outlining the symptoms associated with mild head injury and suggested coping strategies. Those seen only at three months after injury did not receive this booklet.

Results: Patients in the intervention group who were seen at one week and given the information booklet reported fewer symptoms overall and were significantly less stressed at three months after the injury.

Conclusions: The provision of an information booklet reduces anxiety and reporting of ongoing problems.

A controversial issue in the field of head injury is the outcome of mild head injury or concussion. ${ }^{1}$ Most individuals with such injuries are not admitted to hospital and receive a variable degree of medical attention. Symptoms are common in the early days or weeks after injury and include headache, dizziness, sensitivity to noise/light, tinnitus, visual disturbances, restlessness, insomnia, reduced speed of thinking, concentration and memory problems, fatigue, irritability, anxiety and depression. These resolve in many instances. ${ }^{2}$ However, in some cases the difficulties persist, sometimes resulting in significant ongoing adjustment problems. ${ }^{3}$

This variability in outcome has been attributed to numerous aetiological factors. Van Zomeren and van den Burg ${ }^{4}$ proposed that post-concussional symptoms result from the chronic effort required to cope with persisting information processing deficits. Failure to understand the basis of ongoing difficulties may cause frustration and anxiety, exacerbating cognitive difficulties. The development of ongoing problems might, under such circumstances, be curtailed by the provision of information.

Management protocols designed to minimise ongoing problems following mild head injury generally involve the provision of information about such injuries and their effects, a brief screening assessment, and follow up contact. Five studies have examined the impact of such protocols. Studies by Relander et al, ${ }^{5}$ Minderhoud et $a l^{6}{ }^{6}$ and Wade et $a l^{7}$ showed some benefit of intervention in terms of time off work and reported symptoms six months after the injury. On the other hand, studies by Gronwall and by Alves et al showed no significant impact of the provision of information or reassurance upon reported symptoms. ${ }^{89}$ The studies by Relander, Wade, ${ }^{7}$ and Alves ${ }^{9}$ focused on patients admitted to hospital. However, most individuals who sustain uncomplicated mild head injury are not admitted to hospital.

In this study our aim was to establish whether early assessment and the provision of written information and suggested coping strategies could reduce the incidence of these problems in a group of individuals with uncomplicated mild head injuries who were not admitted to hospital.

\section{METHODS}

\section{Participants}

Participants were recruited from consecutive presentations to the emergency departments of two hospitals over a 30 month period. Ethics approval was obtained. Participants were aged 16 years or over, were English speaking, and had a history of trauma to the head resulting in loss of consciousness for less than 30 minutes, post-traumatic amnesia for less than 24 hours, and a Glasgow coma scale (GCS) score of 13-15 on presentation to the emergency department. Participants were recruited only if they had no need for surgery requiring general anaesthesia. Neither computed tomography nor magnetic resonance imaging was undertaken, as the injuries were not considered sufficiently severe. Patients with focal neurological signs were also excluded.

\section{Design and procedures}

Participants with mild head injury were kept under observation in the emergency department, with monitoring of their orientation and memory. When fully oriented, they were discharged home, with standard instructions to contact their local doctor if they experienced further problems. Upon recruitment, which occurred at the end of the observation period, informed consent was obtained.

Participants were alternately assigned to one of two groups. Those assigned to the intervention group were contacted within 48 hours and seen five to seven days after the injury. A detailed history was taken and neuropsychological assessment performed. Intervention group participants were given an information booklet outlining common symptoms associated with mild head injury, their likely time course, and suggested coping strategies. No specific feedback was given regarding the results of the assessments conducted. These individuals were reassessed three months after the injury. Non-intervention control group participants received standard emergency department treatment and were not given the information booklet. They were assessed only at three months after the injury.

\section{Measures}

Intervention group participants completed the measures outlined below at one week and three months postinjury; the non-intervention control group completed the measures at three months only. 
Table 1 Comparison of an intervention group with a non-intervention group after mild head injury with repect to demographic, intelligence, and injury severity variables

\begin{tabular}{llllll}
\hline & \multicolumn{3}{l}{ Mild traumatic head injury } & & \\
\cline { 2 - 4 } Variable & $\begin{array}{l}\text { Intervention } \\
(\mathrm{n}=79)\end{array}$ & $\begin{array}{l}\text { Non-intervention } \\
(\mathrm{n}=123)\end{array}$ & $\mathrm{F}$ & $\mathrm{p}$ Value \\
\hline Age (years) & $24.1(9.1)$ & $28.1(12.2)$ & 6.19 & 0.01 \\
Education (years) & $11.5(1.9)$ & $11.4(1.9)$ & 0.34 & 0.56 \\
SES rank & $4.7(1.1)$ & $4.9(1.0)$ & 1.62 & 0.20 \\
NART FSIQ & $104.6(7.6)$ & $105.1(8.2)$ & 0.13 & 0.71 \\
PTA (min) & $109.8(252)$ & $96.9(154)$ & 0.18 & 0.67 \\
LOC (min) & $4.3(6.4)$ & $4.0(6.3)$ & 0.21 & 0.65 \\
\hline
\end{tabular}

Values are mean (SD).

LOC, loss of consciousness; NART FSIQ, national adult reading test full scale intelligence quotient; PTA, post-traumatic amnesia; SES,

socioeconomic status.

The symptom checklist-90-revised (SCL-90-R) ${ }^{10}$ was used as a measure of psychological adjustment, and the Holmes Rahe survey of recent experiences ${ }^{11}$ as a measure of concurrent life stresses. The post-concussion syndrome checklist (PCSC) $)^{12}$ documented symptoms associated with the injury. Neuropsychological measures of premorbid intelligence quotient, attention, speed of information processing, and memory included the national adult reading test (NART), ${ }^{13}$ four choice reaction time (decision time), the Wechsler adult intelligence scale (reading) (WAIS-R) digit span and digit symbol subtests, the 2.4 and 2.0 second pacings of the paced auditory serial addition task (PASAT), ${ }^{14}$ the speed of comprehension subtest from the speed and capacity of language processing test, ${ }^{15}$ and the Rey auditory-verbal learning test. ${ }^{16}$

\section{RESULTS}

In all, 262 participants with mild head injury agreed to participate in the study-136 in the intervention group and 126 in the non-intervention control group. Of those in the intervention group seen at one week postinjury, 84 participants $(62 \%)$ returned for the three month follow up assessment. Those returning at three months were younger than those who defaulted (mean age $24.1 \vee 30.4$ years; $\mathrm{F}(1)=11.11, \mathrm{p}<0.001)$. There were no significant differences between attenders and non-attenders in socioeconomic status, ${ }^{17}$ duration of post-traumatic amnesia or loss of consciousness, symptoms on the post-concussion syndrome checklist, or neuropsychological test scores at one week.

In order to evaluate the impact of the intervention, our report will focus on participants seen at both one week and three months. Five subjects in the intervention group and three in the non-intervention control group were removed from the analysis as they were outliers. Demographic details, measures of injury severity, and intelligence quotients are set out in table 1. Apart from a significant difference in age $(F(1)=6.19, p<0.01)$, there were no differences between groups in any of these variables, or in terms of history of previous head injury, neurological or psychiatric problems, or learning difficulties. Causes of injury were motor vehicle accident $(27 \%)$, falls $(18 \%)$, cycling accidents $(7 \%)$, assaults (18\%), sporting injuries $(23 \%)$, and other miscellaneous causes $(7 \%)$.

Results obtained at three months in the intervention group were compared with the results in the non-intervention control group using univariate analysis of covariance, with age as a covariate, and $\alpha=0.05$. The non-intervention control group were more likely to report symptoms on the post-concussion syndrome checklist at three months than the intervention group, particularly difficulty in sleeping $(F(1)=6.09$, $\mathrm{p}<0.01$ ), and anxiety $(\mathrm{F}(\mathrm{l})=4.32, \mathrm{p}<0.04)$ (fig 1$)$. The

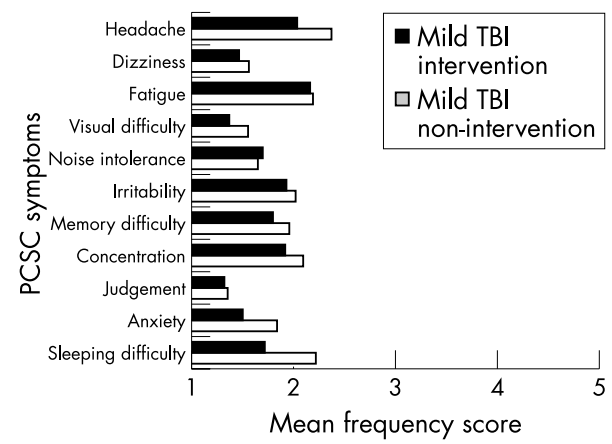

Figure 1 Results of intervention $v$ non-intervention in mild head injury on the post-concussion syndrome checklist at three months after the injury. PCSC, post-concussion syndrome checklist; TBI, traumatic brain injury.

non-intervention mild head injury control group also had higher scores on the symptom checklist global severity index scale $(F(1)=6.74, p<0.01)$, especially the paranoia $(\mathrm{F}(\mathrm{l})=6.25, \mathrm{p}<0.01)$ and hostility subscales $(\mathrm{F}(\mathrm{l})=6.49$, $\mathrm{p}<0.01)$. In summary, subjects in the non-intervention control group reported more symptoms and were significantly more stressed than those in the intervention group.

The two groups performed at a similar level on most neuropsychological measures. Subjects in the intervention group, who had been tested twice, obtained higher (better) scores on the Rey auditory-verbal learning test than the nonintervention group $(\mathrm{F}(\mathrm{l})=21.24, \mathrm{p}=0.000)$. However, evidence from a concurrent study including non-head injured controls, also assessed twice, suggested that an improvement in this score could be a practice effect. ${ }^{18}$

\section{DISCUSSION}

Our results showed that individuals who were not seen at one week after a mild head injury and who did not receive an information booklet with suggested coping strategies reported more symptoms three months later, particularly sleep disturbances and anxiety, than those who did receive these interventions. They also showed higher levels of psychological distress on the symptom checklist-90 R, particularly the hostility and paranoia subscales. These findings suggest that use of the information booklet was beneficial.

Our results are consistent with those of previous reports by Relander et al, ${ }^{5}$ Minderhoud et $a l_{,}{ }^{6}$ and Wade et $a l^{7}$ in which specific instructions regarding management and supportive follow up contact were provided. Those studies did not, however, employ an information booklet. Previous studies that used an information booklet, carried out by Gronwall ${ }^{8}$ and Alves et al, ${ }^{9}$ could not show any significant impact in terms of reported symptoms, although neither of those investigations focused on stress levels.

Our study has certain limitations. The rate of attrition of participants in the intervention group between one week and three months postinjury was quite high, and those who returned were somewhat younger than those who did not. Although there were no differences between attenders and non-attenders for the other variables, it remains possible that those who returned at three months were those with ongoing problems. Despite this, the intervention group still reported fewer problems than the non-intervention control group. It is also possible that the assessment process conducted in the first week after injury was of some therapeutic value, separate from that of the information booklet, although no specific feedback was given to patients at this time regarding the results of the assessment.

A more detailed analysis of symptoms and cognitive difficulties in this study group is the subject of another 
paper. ${ }^{18}$ There is no doubt that numerous factors influence outcome from mild head injury. However, stress levels and poor psychological adjustment appeared to contribute to the maintenance of symptoms. In view of the large number of individuals who sustain mild head injury, it is difficult to justify neuropsychological assessment of all cases. However, the provision of information about such injuries and about suggested coping strategies reduces anxiety and thereby lowers the incidence of ongoing problems to some degree. The information booklet is currently being introduced on a trial basis in the emergency departments of hospitals in the State of Victoria. A copy may be obtained by writing to the senior author.

\section{ACKNOWLEDGEMENTS}

This research was funded by a grant from the Ministerial Implementation Committee on Head Injury, Health Department of Victoria, Australia.

\section{Authors' affiliations}

J Ponsford, C Willmott, R Nelms, C Curran, Monash-Epworth Rehabilitation Research Centre, Epworth Hospital, Richmond, Victoria, Australia

A Rothwell, Julia Farr Services, Fullarton, Australia

P Cameron, Royal Melbourne Hospital, Melbourne, Australia

A-M Kelly, Western Hospital, Melbourne, Australia

Competing interests: none declared.

Correspondence to: Associate Professor J Ponsford, Department of Psychology, Bethesda Rehabilitation Centre, Epworth Hospital, 30 Erin Street, Richmond, Victoria 3121, Australia;

jennie.ponsford@med.monash.edu.au

Received 16 June 2001

In revised form 4 April 2002

Accepted 18 April 2002

\section{REFERENCES}

1 Berrol S. Terminology of post-concussion syndrome. In: Horn L, Zasler N, eds. Rehabilitation of post-concussive disorders. Physical medicine and rehabilitation: state of the art reviews, vol 6. Philadelphia: Hanley and Belfus, 1992: 1-8

2 Dikmen S, McLean A, Temkin N, et al. Neuropsychological and psychosocial consequences of minor head injury. J Neurol Neurosurg Psychiatry 1986;49:1227-32.

3 Gronwall D. Minor head injury. Neuropsychology 1991;5:253-65.

4 van Zomeren $\mathbf{A H}$, van den Burg W. Residual complaints of patients two years after severe head injury. I Neurol Neurosurg Psychiatry 1985:48:21-8.

5 Relander M, Troupp H, Bjorksteinaf G. Controlled trial of treatment for cerebral concussion. BM 1972;iv:777-9.

6 Minderhoud JM, Boelens MEM, Huizenga J, et al. Treatment of minor head injuries. Clin Neurol Neurosurg 1980;82:127-40.

7 Wade DT, King NS, Wenden FJ, et al. Routine follow up after head injury: a second randomised controlled trial. J Neurol Neurosurg Psychiatry 1998;65:177-83.

8 Gronwall D. Rehabilitation programs for patients with MHI: components, problems and evaluation. J Head Trauma Rehabil 1986;1:53-62.

9 Alves W, Macciocchi SN, Barth JT. Postconcussive symptoms after uncomplicated MHI. J Head Trauma Rehabil 1993;8:48-59.

10 Derogatis LR. Symptom checklist SCL-9O-R: administration scoring and procedures manual - I. Baltimore: Clinical Psychometric Research, 1977.

11 Holmes TH, Rahe RH. The social readjustment rating scale. J Psychosom Res 1967:11:213.

12 Gouvier WD, Cubic B, Jones $G$, et al. Postconcussion symptoms and daily stress in normal and head-injured college populations. Arch Clin Neuropsychol 1992;7:193-211.

13 Nelson HE, Willison J. National adult reading test (NART): test manual, 2nd ed. Windsor: NFER Nelson, 1991.

14 Gronwall DMA, Sampson H. The psychological effects of concussion. Auckland: Auckland University Press, 1974.

15 Baddeley A, Emslie H, Nimmo-Smith I. The speed and capacity of language (processing test: manual). Bury St Edmunds: Thames Valley Test Company, 1992

16 Rey A. L'Examen clinique en psychologie. Paris: Presses Universitaires de France, 1964

17 Daniel A. Power, privilege and prestige: occupations in Australia. Melbourne: Longman-Cheshire, 1983.

18 Ponsford J, Willmott C, Rothwell A, et al. Factors influencing outcome following mild traumatic brain injury in adults. J Int Neuropsychol Soc 2000;6:568-79. 